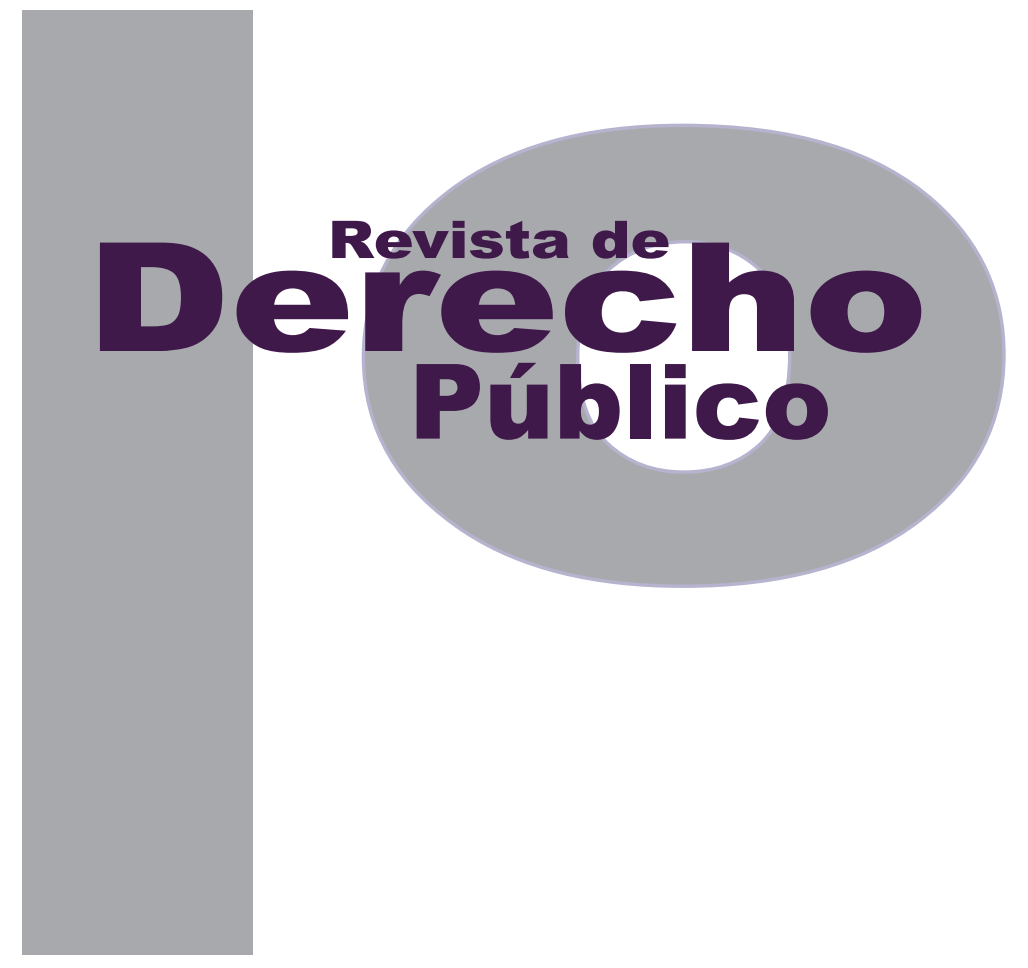

\title{
LA PARTICIPACIÓN DE LOS PUEBLOS ÉTNICOS EN LA NEGOCIACIÓN DEL FIN DEL CONFLICTO ARMADO COLOMBIANO
}

\author{
GRUPO COPAL \\ Artículo de reflexión \\ DOI: http://dx.doi.org/10.15425/redepub.33.2014.20 \\ Universidad de los Andes \\ Facultad de Derecho \\ Revista de Derecho Público N. ${ }^{\circ} 33$ \\ Julio - Diciembre de 2014. ISSN 1909-7778
}




\title{
La participación de los pueblos étnicos en la negociación del fin del conflicto armado colombiano
}

\section{Resumen}

El proceso de paz iniciado entre el gobierno de Juan Manuel Santos y las Fuerzas Armadas Revolucionarias de Colombia - Ejército del Pueblo (FARC-EP), en noviembre de 2012, motivó este análisis sobre la participación que deben tener los pueblos étnicos de Colombia en la negociación y construcción de la paz, desde su propia visión. El conflicto armado interno ha generado graves afectaciones al interior de estos pueblos al vulnerar sus derechos colectivos e individuales, frente a los cuales es posible invocar la efectividad del mecanismo de participación y derecho fundamental de la Consulta Previa, mecanismo que ha sido reconocido como el más importante para combatir la exclusión de las comunidades. Con este propósito, en la primera parte se presenta la situación de los pueblos étnicos como víctimas diferenciadas en el marco del conflicto armado y el desplazamiento forzado, con soporte en pronunciamientos judiciales; en la segunda parte se expone el rol que estos deben ejercer ante las posibilidades de construcción de una paz con legitimidad; por esta vía, la tercera parte describe el derecho fundamental a la Consulta Previa en el derecho nacional e internacional; y en la última parte se observa la Consulta Previa en el marco del proceso de paz desde la óptica de los afectados.

Palabras clave: Consulta Previa, pueblos étnicos, participación política, autodeterminación, paz, referendo, Asamblea Nacional Constituyente.

\section{The participation of ethnic people in Colombian peace process}

\begin{abstract}
The peace talks initiated between the government of Juan Manuel Santos and the guerrilla FARC EP, in November of 2012, inspired us to question the role that ethnic peoples should have in the development of these talks, and in the later peace building process. The latter, given that the conflict itself has had deep consequences in the lives of ethnic peoples, undermining their collective and individual rights. Both, international and national law, have recognized their right to participate in any decision that could have any affect upon them, in order to combat the exclusion of these communities. For this purpose, the first part of this text,, tries to illustrate the situation of ethnic groups as differentiated victims in armed conflict, later on, we will question the roll that this groups should have in order to allow the construction of peace with legitimacy. Finally this paper will analyze the right of participation found in prior consultation, in both national and international law and the way this could possibly affect the construction of peace.
\end{abstract}

Keywords: consultation, ethnic groups, political participation, self-determination, peace, referendum, national constituent assembly.

\section{A participação dos povos étnicos no processo de paz}

\section{Resumo}

O processo de paz iniciado entre o governo de Juan Manuel Santos e as Forças Armadas Revolucionárias da Colômbia - Exército Popular (FARC-EP), em novembro de 2012, motivou esta análise sobre a participação que devem ter os povos étnicos da Colômbia na negociação e construção da paz, desde sua própria visão. O conflito armado interno tem gerado graves afetações no interior destes povos ao vulnerar seus direitos coletivos e individuais, frente aos quais é possível invocar a efetividade do mecanismo de participação e direito fundamental da consulta prévia, mecanismo que tem sido reconhecido como o mais importante para combater a exclusão das comunidades. Com este propósito, na primeira parte é apresentada a situação dos povos étnicos como vítimas diferenciadas no marco do conflito armado e o deslocamento forçado, com suporte em pronunciamentos judiciais; na segunda parte é exposto o papel que estes devem exercer ante as possibilidades de construção de uma paz com legitimidade; por esta via, a terceira parte descreve o direito fundamental à consulta prévia no direito nacional e internacional; e na última parte é observada a consulta prévia no marco do processo de paz desde a ótica dos afetados.

Palavras-chave: consulta prévia, povos étnicos, participação política, autodeterminação, paz, referendo, Assembleia Nacional Constituinte. 


\title{
La participación de los pueblos étnicos en la negociación del fin del conflicto armado colombiano*
}

\author{
Grupo COPAL**
}

\section{SUMARIO}

Introducción - I. LOS PUEBLOS ÉTNICOS SON VÍCTIMAS DEL CONFLICTO - A. El auto 004: los pueblos indígenas - B. El auto 005: Ios pueblos afrocolombianos - II. PROPUESTAS PARA LA LEGITIMACIÓN - III. LA CONSULTA PREVIA - IV. LA CONSULTA PREVIA EN EL PROCESO DE PAZ, DESDE LA ÓPTICA DE LOS AFECTADOS - IV. CONCLUSIONES - Referencias.

* Cómo citar este artículo: Grupo COPAL. (Diciembre, 2014). La participación de los pueblos étnicos en la negociación del fin del conflicto armado colombiano. Revista de Derecho Público, 33. Universidad de los Andes (Colombia).

** Colectivo de Estudios Poscoloniales/Decoloniales en América Latina, correo: coepal@gmail.com. Pertenecen: Laura Natalia Gil Vega (Ingilv@unal.edu. co) y Diana Marcela Ricaurte Vargas (dmricaurtev@unal.edu.co), estudiantes de Derecho, Universidad Nacional de Colombia; Jennifer Andrea Montano Granados, abogada y estudiante de la Maestría en Derecho, profundización en Derecho Penal, Universidad Nacional de Colombia (jamontanog@unal.edu. co); Laura María Guevara Agudelo, filósofa Universidad Nacional de Colombia, estudiante de Derecho Universidad de los Andes, Colombia (Imguevaraa@ unal.edu.co, Im.guevara79@uniandes.edu.co). 
Introducción

El grupo de investigación COPAL $^{1}$ de la Universidad Nacional de Colombia, Facultad de Derecho y Ciencias Políticas, en desarrollo del proyecto de investigación sobre la Consulta Previa respecto a la negociación del conflicto armado que se adelanta en La Habana, se cuestionó y se propuso analizar la pertinencia, obligatoriedad y necesidad de determinar en él un espacio de participación para los pueblos étnicos, ya fuera por medio de los mecanismos de participación propuestos en su tiempo por la mesa de negociación, o por medio del derecho fundamental y mecanismo de participación constituido para esta población, esto es la Consulta Previa, considerado como el más idóneo para permitir que estos pueblos participen en las decisiones que les afecten.

Lo anterior, bajo la comprensión de que el conflicto armado no solo ha constituido un factor de grave afectación y vulneración de todos sus derechos, sino que también ha conllevado un riesgo de exterminio físico y cultural, y que el cese

1 El Colectivo de Estudios Poscoloniales/Decoloniales en/de América Latina (Grupo COPAL) es un grupo de investigación de la Facultad de Derecho, Ciencias Políticas y Sociales, adscrito a la Unidad de Investigaciones Jurídico-Sociales "Gerardo Molina" (unIJus). Fue creado en 2006, por iniciativa de estudiantes y docentes interesados/as en el análisis interdisciplinario sobre problemáticas de la sociedad y del sistema-mundo vinculados con: la constitución de subjetividades desde el discurso jurídico, el contenido y alcance de derechos étnicos, así como las propuestas interculturales para la articulación de mecanismos democráticos dentro del Estado social de derecho. Para tal efecto, el Grupo copal analiza de manera crítica la relación entre la sociedad y los discursos jurídicos. Lo anterior, teniendo como base los métodos que ofrecen las teorías poscoloniales y decoloniales de origen latinoamericano; el diálogo permanente entre el multiculturalismo y la interculturalidad, así como la analítica del poder. Por ello, es posible describir al grupo como un caleidoscopio que procura abordar problemas de la sociedad y del derecho a partir de los sujetos políticos que emergen "desde abajo". de este a través de una posible negociación implicaría la toma de decisiones sobre temas de trascendental importancia para su supervivencia y derechos, erigiéndose de esta manera una obligación en cabeza del Estado consistente en asegurar su participación por medio de mecanismos que permitan garantizar su permanencia física y cultural en la sociedad, y la construcción real de paz en la sociedad como sujetos activos de esta.

Es por ello que el Grupo copal estimó necesario recurrir al análisis de la Consulta Previa, así como a la práctica de entrevistas a integrantes de pueblos étnicos y de sus organizaciones representativas, para conocer su percepción frente a dicho derecho fundamental y su posible aplicación respecto a las decisiones que se tomen como fruto de la negociación del conflicto armado. Lo anterior, por estimar que son ellas y ellos los más indicados para conceptuar acerca de la cuestión que nos convoca, además de recurrir a personas que no se autorreconocen como integrantes de pueblos étnicos, pero cuyo transcurrir profesional las ha involucrado en el conocimiento de los procesos de participación de este tipo.

Para esto optamos por el acercamiento a través de conversaciones en torno a temas específicos, aunque no de manera inflexible ni estandarizada. En esa medida nos preocupamos por ofrecerles amplia libertad en la estructuración de sus entrevistas, alejándonos lo más posible de un esquema de encuesta, pues el objetivo era escucharlas y entender sus puntos de vista, lo cual nos aproximaría a la realidad en deter- 
minados ámbitos (Corbetta, 2007). Así pues, la entrevista semiestructurada fue la metodología elegida; en ella el entrevistador dispone de un guión que recoge, sin un orden preestablecido, los temas que se van a abordar, lo que posibilita una mayor fluidez en el desarrollo de la conversación (Corbetta, 2007).

Por tratarse de una entrevista cualitativa, las personas no fueron escogidas con la pretensión de llegar a muestras distintivas en sentido estadístico, sino atendiendo a su calidad de sujetos representartivos de tres grupos seleccionados: en primer lugar, autoridades o líderes de los pueblos étnicos que dada su trayectoria política, laboral y su experiencia con las organizaciones nos permitieran conocer las posturas construidas al interior de sus pueblos y procesos organizactivos; en segundo lugar, integrantes del Gobierno nacional conocedores del tema de Consulta Previa desde el ámbito institucional; finalmente investigadores/as y académicos/as que pudieran aportar sus puntos de vista.

En el primer grupo tuvimos la oportunidad de entrevistar a Axcan Duque, abogado especialista en derechos humanos, quien para el momento de la entrevista era asesor jurídico de AFRODES (Asociación de Afrocolombianos Desplazados); a José Ben Hur Teteye Botyay, secretario general de la OPIAC (Organización de los Pueblos Indígenas de la Amazonía Colombiana). En la Organización Nacional Indígena de Colombia (onic) dialogamos con Feliciano Valencia Medina, indígena nasa del Cauca, autoridad de importante incidencia en el movimiento indígena nacional; Juvenal Arrieta González, indígena del pueblo embera chamí, secretario general de la onıc; Luis Evelis Andrade Casamá, quien fue consejero mayor de la organización y actualmente senador de la República por la circunscripción especial; María Deysi, indígena nasa con quien compartimos en las instalaciones de la organización a propósito de su trabajo político con las mujeres de su comunidad; y finalmente Rodolfo Adán Vega Luquez, indígena Kankuamo, asesor general del Consejo Mayor de Gobierno de la onic.

En el segundo grupo entrevistamos al director de Consulta Previa del Ministerio del Interior, Álvaro Echeverry Londoño, abogado especializado en derecho administrativo. Del grupo de académicos contamos con los aportes de Clara Inés Henao, profesora de la Facultad de Ciencias Humanas de la Universidad Nacional de CoIombia y docente del Diplomado sobre Consulta Previa que se realizó en esta institución en el año 2013.

Parte de los testimonios son recogidos en este artículo para ilustrar con más certeza el asunto que nos ocupa. La primera parte presenta la situación de los pueblos étnicos como víctimas diferenciadas en el marco del conflicto armado y el desplazamiento forzado, con soporte en pronunciamientos judiciales. La segunda expone el rol que estos deben ejercer ante las posibilidades de construcción de una paz con legitimidad. Por esta vía, la tercera parte describe el derecho fundamental a la Consulta Previa en el derecho nacional e internacional, para, en la última parte, observar la Consulta Previa en el marco del proceso de paz desde la óptica de los afectados. 


\section{LOS PUEBLOS ÉTNICOS SON VÍCTIMAS DEL CONFLICTO}

Más de cincuenta años de violencia han traído innumerables consecuencias sobre la población colombiana. El conflicto que ha tenido que afrontar nuestro país se ha caracterizado por ser un fenómeno de larga duración, variable intensidad y gran complejidad (Restrepo y Aponte, 2009) que ha dejado aproximadamente 6 043473 víctimas entre 1984 y 2013 (Semana, 2014).

La sociedad civil ha sufrido de manera profunda los efectos de la guerra. Desde mediados de los años 80 la cifra de desplazados por el conflicto armado supera los 5000000 de personas, dentro de las que según los informes de la ONG Amnistía Internacional se encuentran en su mayoría campesinos, indígenas y afrodescendientes. La violencia no es la misma en todo el país, y esta es otra característica trascendental del conflicto armado interno en Colombia; los datos confirman que ha sido más cercana a grupos indígenas y afrodescendientes situados principalmente en el Cauca y el Valle del Cauca: en lo que va corrido del 2014 se han presentado 3192 eventos de vulneraciones de derechos humanos contra pueblos indígenas, de los cuales 10 han sido homicidos selectivos contra sus autoridades (onIC, 2014).

Hablar de los efectos del conflicto armado interno sobre los pueblos étnicos que conviven en el territorio colombiano implica no solo asumir que son víctimas individuales que hacen parte de las desastrozas cifras que este ha registrado a lo largo de su historia, sino que resulta importante referirnos a su victimización desde una visión colectiva que ha trastocado su identidad y cosmovisión. En las entrevistas realizadas a integrantes de pueblos indígenas y afrodescendientes, líderes y conocedores de la situación que estos afrontan por el conflicto armado interno, se destacó la importancia de la participación de los pueblos étnicos en la negociación del fin del conflicto armado y un posible escenario de paz. Para ellos surge necesario abrir un espacio de participación en el proceso de paz que se adelanta entre el Gobierno y las FARC-EP, principalmente en virtud de la afectación diferencial que han sufrido a causa del conflicto armado. Sobre esta forma de afectación Rodolfo Adán Vega Luquez manifiesta:

La afectación no solo ha sido en mi persona sino a toda una comunidad, a todo un territorio, a unos sitios sagrados, a toda mi identidad cultural que es donde nosotros vivimos armónicamente en equilibrio con el mundo natural, por ende la victimización que se ha hecho no solo afecta a una persona sino a todo un pueblo, a todo un territorio. De hecho, cuando discutíamos el tema del decreto ley de víctimas para comunidades indígenas, uno de los artículos que quedó es que para los pueblos indígenas el territorio es considerado como víctima, entonces nosotros no nos creemos dueños del territorio sino parte de él, esa es la diferencia con quienes no tienen esa cosmovisión, esa forma de ver el mundo.

Como un logro legislativo, los pueblos han resaltado la importancia de la Ley de Víctimas 
(Ley 1448 de 2011), que en el artículo 3 define como víctimas las "personas que individual o colectivamente hayan sufrido un daño por hechos ocurridos a partir de 1985, como consecuencia de infracciones al derecho internacional humanitario o de violaciones graves y manifiestas a las normas internacionales de derechos humanos, ocurridas con ocasión del conflicto armado interno".

Esta noción de víctima incluye la visión colectiva del daño de los pueblos étnicos, quienes no solo se reconocen como víctimas individuales sino también como parte integral de un sujeto colectivo de derechos en riesgo de exterminio físico y cultural como consecuencia del conflicto. A algunos integrantes de pueblos indígenas afectados les resulta importante resaltar no solo su calidad de víctimas diferenciadas por pertenecer a un grupo protegido de manera colectiva, sino la importancia de su victimización en la historia. En palabras de Rodolfo Adán Vega Luquez: "hemos sido víctimas de este conflicto atroz que vive Colombia desde hace más de 500 años, y en mi caso desde que nací, porque los pueblos indígenas han sido afectados por situaciones externas de violencia, de guerra, que hemos vivido en nuestros territorios y nuestros pueblos."

De igual manera, su condición de víctimas diferenciadas incluye un efecto particular que involucra a los integrantes de estos pueblos con sus territorios, los cuales son considerados como sujeto vivo e, igualmente, víctima del conflicto. Recordemos que la base social y cultural de sujetos colectivos y étnicos es su estrecha relación con el espacio que habitan, el cual, como con- secuencia de los enfrentamientos y de algunas políticas económicas se ha visto afectado, conduciéndolos al exterminio físico y cultural:

si nosotros pensamos en el territorio como la fuente de vida, como la que suministra el pan, como la que da todo en la vida, entonces ahí tendríamos otro tipo de afectación, y cuando consideramos que los ríos son los principales actores fluviales de nuestra comunicación, los confinamientos y los desplazamientos forzados fueron destruyendo un poco esos aspectos culturales que nosotros teníamos y que no se pueden reproducir de la misma forma en los otros territorios (Axcan Duque).

La vulneración colectiva e histórica de los derechos de los pueblos étnicos en Colombia ha prendido las alarmas de las autoridades locales y de la comunidad internacional, demandando urgente atención a la grave situación que afrontan las comunidades en el contexto del conflicto armado y que ha dejado catastróficas consecuencias. El monitoreo anual emprendido por la Consultoría para los Derechos Humanos y el Desplazamiento (codHEs, 2012) arrojó un total de 73 desplazamientos masivos, que incluyeron 29.521 personas obligadas a dejar sus hogares por causa de los enfrentamientos entre los grupos armados al margen de la ley, de estos contra la fuerza pública y por operaciones de erradicación de cultivos ilícitos. Los cinco departamentos más afectados fueron en su orden: Cauca (8.829 personas, en 31 desplazamientos), Córdoba (3.679 personas, en 10 desplazamientos), Nariño (4.735 personas, en 10 desplazamientos), Antioquia (9.373 personas, en 6 desplazamientos), Chocó (994 personas, 
en 6 desplazamientos), Valle del Cauca (1.425 personas, en 4 desplazamientos).

La preocupación que existe en torno a la situación de los pueblos étnicos ha llevado a organizaciones indígenas y afrodescendientes a demandar ante entidades internacionales protección de sus derechos colectivos e individuales vulnerados por el conflicto. El 14 de marzo de 2013, la onIC presentó ante la Organización de los Estados Americanos (OEA) un informe sobre la situación de los pueblos indígenas que se sienten vulnerables debido a la violencia, el desplazamiento, la discriminación, la pobreza y el abandono institucional por parte del Gobierno colombiano. El informe destaca la situación crítica de más de 66 pueblos indígenas que enfrentan el exterminio físico y cultural, muchos de los cuales tienen una población de menos de 500 personas. Su vida se ve afectada por las operaciones militares y las concesiones mineras en sus territorios, que en algunos casos totalizan el 54 por ciento de los resguardos indígenas (oNIC, 2013.

La Corte Constitucional de Colombia, ante la situación crítica en la que se encuentran estos pueblos y a propósito de la declaración del "estado de cosas inconstitucional", realizada mediante los autos de seguimiento a la sentencia T-025 de 2004, ha permitido la caracterización fáctica de las múltiples afectaciones y riesgos que existen para los pueblos étnicos en el marco del conflicto armado y el desplazamiento forzado por medio de varias providencias.

Teniendo en cuenta la competencia jurídica que la Corte mantiene mientras no se restablezcan los derechos fundamentales o se eliminen las causas de su amenaza a la población desplazada étnicamente diferenciada, esta corporación expidió en el 2009 dos autos de seguimiento de gran trascendencia: el auto 004 y el auto 005. El primero está referido a la constatación y descripción de la situación humanitaria de los pueblos indígenas, mientras que el segundo hace lo propio con relación a los pueblos afrodescendientes. Este apartado resume el análisis hecho por la Corte Constitucional y argumenta que los derechos diferenciales y las afectaciones agudas reconocidas judicialmente evidencian la exclusión sistemática de los pueblos étnicos de la mayoría de ámbitos sociales que permitirían su pervivencia física y permanencia cultural, lo cual podría repercutir en un posible escenario de paz.

\section{A. El auto 004: los pueblos indígenas}

El análisis fáctico realizado por la Corte Constitucional en relación con los pueblos indígenas describe las principales afectaciones de que son víctimas, con base en las intervenciones realizadas por sus organizaciones, diferentes sectores de la sociedad civil y el Gobierno. De conformidad con el estudio de la Corte se pueden identificar dos tipos de factores: unos de afectación directa que pueden o no involucrar activamente a los pueblos indígenas y a sus integrantes en el conflicto armado, y otros que los incluyen en procesos vinculados y subyacentes relacionados con procesos territoriales y socioeconómicos. En estos últimos es posible distinguir aqueIlos elementos que perjudican directamente los 
territorios y la cultura indígena, y aquellos que de manera indirecta se ven exacerbados con ocasión de la guerra.

La Corte constató estos factores en la situación concreta de 34 pueblos indígenas, y concluyó afirmando que estos se encuentran en "grave riesgo de exterminio físico y cultural”. En razón a ello ordenó la creación de un programa de garantía de los derechos y de planes de salvaguarda étnica, en desarrollo de lo cual se crearon medidas específicas para cada pueblo indígena así como las órdenes de protección especial para personas individualmente consideradas.

Posteriormente fue incluido dentro de estas medidas el pueblo Hitnú o Macaguán asentado en el departamento de Arauca. Así mismo, ante la permanencia de la vulneración de derechos del pueblo Awa, asentado en Nariño y Putumayo, la Corte adoptó medidas de carácter específico sin precedentes en el seguimiento efectuado: la adopción de un plan provisional urgente de reacción y contingencia que debía contar con Consulta Previa y con presupuesto propio mediante la inscripción en el Banco de Proyectos de Inversión Nacional, y la aceleración del Plan de Salvaguarda Étnica. Sin embargo, a pesar del activismo de la Corte y de la especificidad de su intervención en las políticas públicas, los informes de las organizaciones reflejan un alto grado de incumplimiento de sus órdenes y la no superación de la crisis humanitaria para los pueblos indígenas (Rodríguez, Alfonso y Cavelier, 2009).
B. El auto 005: los pueblos afrocolombianos

Las constataciones realizadas por la Corte Constitucional respecto a los pueblos afrocolombianos se sostienen sobre la necesidad de implementar un enfoque diferencial que reconozca su diversidad. En efecto, para la guardiana de la Constitución el alto subregistro y la precariedad de la información no permiten de forma certera caracterizar a la población desplazada y menos aún tener en cuenta diferencias en razón de su pertenencia étnica. No obstante, ello no releva al Estado de las obligaciones internacionales adquiridas frente a los pueblos afrodescendientes, dentro de las cuales se encuentra la observancia del Convenio 169 de la Organización Internacional del Trabajo (оІт); por el contrario, advierte sobre la urgencia de materializar los derechos de estos como sujetos de especial protección constitucional y protección reforzada.

Basada en la información suministrada por los intervinientes en el marco del proceso de seguimiento a la sentencia T-025 de 2004, la Corte señala algunas particularidades del desplazamiento de los pueblos afrocolombianos: la violencia rural y urbana asociada al control territorial, el predominio de desplazamientos intraurbanos e intraveredales, la persistencia del conflicto armado en los territorios ancestrales y la resistencia de la población arraigada en el territorio. Igualmente, tres tipos de factores transversales que inciden en el desplazamiento: las presiones generadas por procesos mineros y agrícolas, la desprotección jurídica de los territorios colectivos y la exclusión estructural que his- 
tóricamente les afecta. Todos estos factores se entrecruzan y producen mayores riesgos para los derechos de los pueblos afrocolombianos.

Dentro de estos riesgos la descripción de la Corte identifica diez tipos de facetas del desplazamiento que pueden agravar la situación de la población afrodescendiente. El estudio de estas facetas se basa en los casos presentados por los intervinientes, con base en los cuales se detecta que la situación es más grave en aquellos municipios y ciudades de población mayoritariamente afrodescendiente, residente en territorios colectivos, desplazada o confinada.

Si bien los pueblos étnicos son víctimas del conflicto armado, y su situación ha sido denunciada por la comunidad internacional a través de diferentes organizaciones así como por la misma Corte Constitucional de Colombia y otras autoridades, resulta aún más significativo entender que la situación de víctimas tiene un contenido colectivo y étnico. En el desarrollo del conflicto armado estos pueblos han sido víctimas de afectaciones que los involucran directamente, como señalamientos, asesinatos, amenazas, confinamientos, control sobre su movilidad, reclutamiento, pérdidas territoriales y pérdida de soberanía sobre los territorios, entre otras formas de violencia cuyo contenido también transgredió la identidad que por años han intentado proteger, y cuyos elementos están circunscritos a la cosmovisión colectiva y cultural propia de cada pueblo y de sus territorios.

El inicio de los diálogos entre el gobierno del presidente Juan Manuel Santos (2010-2014) y la guerrilla de las FARC-EP a mediados de 2012 abre una vez más la esperanza del fin del conflicto y el inicio de la paz en Colombia. Sin embargo, el camino para alcanzar dichos propósitos suscita la pregunta por el papel que reclaman los pueblos étnicos como propietarios de parte del territorio en disputa dentro del conflicto armado interno. Los evidentes efectos que ha tenido el conflicto en la vida de diferentes individuos permiten cuestionarnos sobre el rol que estos deben ejercer en la posible construcción de la paz, a partir de una justicia que transforme y supere las lógicas de la discriminación histórica.

\section{PROPUESTAS PARA LA LEGITIMACIÓN}

El líder indígena Feliciano Valencia afirmó al respecto, que el proceso de paz debe contar con medidas que no surjan de decisiones unilaterales de parte del Estado o que involucren solo a quienes hoy se sientan en La Habana, pues esto no constituiría una verdadera construcción de paz. De acuerdo con los entrevistados, lo que se debería buscar por medio del diálogo es lo que ellos denominan "reconciliación ciudadana", algo en lo que tanto los pueblos étnicos como la sociedad deben participar para evitar la continuidad de la violencia.

A esta perspectiva, Luis Evelis Andrade agregó que el proceso de paz, al integrar la participación de los pueblos étnicos y de toda la sociedad, debería tener una dimensión pedagógica, "en la cual las víctimas sean los principales actores de construcción de paz y de superación 
del conflicto social que se genera como consecuencia del conflicto armado". Vale resaltar que para esta autoridad, así como para muchos de los entrevistados, la intervención de la sociedad en la construcción de la paz, se dé o no dentro del proceso que se adelanta hoy, no debe estar dirigida exclusivamente a decisiones en torno a la justicia en términos penales, sino en términos de inclusión y reconocimiento de diversidad y diferencia, pues solo así se superan los problemas estructurales que originan la violencia.

La necesidad de involucrar a la sociedad en los acuerdos pactados en La Habana ha sido un reclamo notorio. Distintos sectores de la sociedad han manifestado la importancia de que los colombianos que no se encuentran representados en la mesa de diálogo de La Habana tengan una participación activa en la negociación del fin del conflicto armado y un posible escenario de paz. Ante las críticas a la falta de participación, el Gobierno ha propuesto la realización de un referendo para legitimar lo allí decidido y que sean los colombianos y colombianas quienes tomen las decisiones en última instancia. Por su parte, las FARC-EP son partidarias de una Asamblea Nacional Constituyente que permita modificar el orden jurídico y social de manera acorde con sus propuestas de orden institucional.

Frente a los mecanismos de participación propuestos desde la mesa de negociación para la refrendación de los acuerdos, se puede afirmar que la mayoría de los integrantes de pueblos étnicos entrevistados tienen una posición adversa y crítica, pues tanto la Asamblea Nacional Constituyente como el referendo son vistos como instrumentos ineficaces para la garantía de sus derechos.

Sobre la Asamblea Nacional Constituyente, Axcan Duque afirma que no sería un mecanismo que satisfaga las necesidades de participación que tiene el pueblo Afrocolombiano, basado en la desconfianza que le inspira la Constitución de 1991: "apuesto que en la constituyente tampoco van a estar nuestras afectaciones ni nuestras necesidades como grupo étnico, o si no, pregúntenle a la Constitución de 1991, que como la única y gloriosa Constitución en el mundo pone artículos transitorios, entre esos el 55 que ahí quedamos nosotros para la Ley $70 . "$

Para varios integrantes de la onIc, así como para Axcan, la Asamblea Nacional Constituyente genera miedos y desconfianzas, pues es vista como un salto al vacío en la medida en que se pone en riesgo el reconocimiento de ciertos derechos alcanzados tímidamente en la Constitución de 1991. Por ejemplo, Luis Evelis Andrade teme a lo que llama "una constituyente sin garantías", y Juvenal Arrieta manifestó que existe el temor para los pueblos de estar en desventaja por ser parte de aquella sociedad que no ostenta el poder político, además de encontrarse dividida en su interior. En esa medida, entiende que difícilmente serían tenidos en cuenta ante una nueva Constitución que derogue la anterior, como sucede con la absorción que sufre la visión de desarrollo de los pueblos indígenas frente a la visión mayoritaria. Estas fueron sus palabras:

Para mí particularmente una constituyente es un salto al vacío porque Colombia es un país 
derechizado y los referentes de opinión son netamente mediáticos... creo que se neoliberalizaría más la Constitución, además estaríamos en una Asamblea Nacional Constituyente en una total desventaja (...) los sectores populares sí existimos, pero no tenemos la capacidad de cohesión tan fuerte para codearnos de codo a codo con aquellos que sí saben manejar el poder para definir las reglas del juego desde la sociedad que nos tocó.

Y agregó,

en este momento una Asamblea Nacional Constituyente no es pertinente y no porque las asambleas constituyentes sean malas, yo creo que, yo le escuché una vez a alguien que leía a Rosseau y a los contractualistas que las asambleas más allá que ser constituyentes, son pactos de paz que pactan las reglas de convivencia (...) en Colombia, las situaciones del derecho se ven desbordadas por dinámicas de poder, de forma atomizada y concentrada y más allá de la cuestión de las dinámicas de poder bajo un referente de identidad netamente criminal violento, entonces las aspiraciones, las reglas de juego, las nociones o los imaginarios que hay en los diferentes grupos de la sociedad muchas veces se constituyen no alrededor de la moral o de la ética del derecho, sino de las armas y el control militar que tiene que ver con otro tipo de cosas que a la vez son el motor del conflicto.

Sobre el mecanismo de referendo propuesto por el Gobierno opinó que este es un sofisma de distracción de utilidad momentánea, que no implicaría un verdadero acuerdo político de voluntades entre la sociedad civil, que es la forma como él considera que deben hacerse los acuerdos en el proceso de paz.
Otros entrevistados coinciden con Juvenal Arrieta en cuanto a que el referendo no permite una real participación o construcción de paz: "a mí lo que me preocupa de la refrendación es que solo le pregunten a los colombianos: usted está de acuerdo con esto que se acordó, ¿sí o no? ... entonces nosotros no estamos siendo parte activa en la construcción de esos acuerdos y eso es lo que estamos reclamando los pueblos indígenas" (Rodolfo Adán Vega).

Se encontró, igualmente, que algunos integrantes de pueblos étnicos entrevistados si bien no se oponen tajantemente a estos mecanismos de participación, sí amoldan su posición. Tanto Feliciano Valencia como Rodolfo Adán hacen una diferencia sobre las etapas del proceso de paz. Para Valencia las modificaciones que surjan como consecuencia de los acuerdos requieren la realización de una Constituyente, sin embargo esta no sería suficiente para la segunda fase o la implementación de los acuerdos, en la cual serían necesarios mecanismos con alcances y efectos distintos:

Después viene la parte de implementación de los acuerdo que ahí es donde venimos a jugar nosotros, que las farc y el Gobierno nos expliquen a nosotros cómo van a implementar estos acuerdos, ¿lo van hacer bajo qué figura?, ¿plan de desarrollo nacional?, ¿con Conpes?, ¿con presupuesto?, ¿cómo lo van a implementar? Segundo: ¿qué van a implementar, proyectos productivos?, ¿asociaciones cooperativas? No sabemos, y si fueran proyectos a gran escala dónde vamos a estar involucrados nosotros, es ahí donde juega la consulta previa. 
Por su parte, Rodolfo Adán manifiesta que la construcción de paz no se puede identificar con la terminación del conflicto. La primera es un proceso mucho más complejo que requiere la participación activa de los pueblos, mientras el segundo se da solo entre los actores del conflicto sentados en La Habana, que incluso podría producirse mediante alguno de los mecanismos propuestos, pero que no debe decidir, por la escasa participación de las víctimas y de la sociedad en general, el futuro de Colombia. Con respecto a esto Juvenal Arrieta indicó:

saludamos que estas iniciativas marquen una ruta hacia la desmovilización de las FARC, y por supuesto una esperanza hacia la paz, pero creemos que las reglas del juego que nos deben satisfacer son las que se construyan con todos nosotros, no solo con indígenas, sino con los sectores de la sociedad, porque la paz se hace es en la sociedad, no entre armados, ellos armarán los acuerdos de desarme, de no utilizar más el fusil, pero la reconciliación, la consciencia de paz, la capacidad de perdonar está en los corazones de los ciudadanos.

Finalmente, se tiene que los entrevistados fueron más allá de los mecanismos propuestos desde La Habana, pues no se limitaron a exponer sus críticas a estos, sino que plantearon fórmulas mucho más complejas. Por ejemplo, José Ben Hur Teteye propuso foros nacionales o departamentales tanto para los pueblos étnicos como para el resto de la sociedad, ante la necesidad de que toda Colombia participe, y al mismo tiempo manifestó su total apoyo a las propuestas de Asamblea Nacional Constituyente o referendo, en la medida en que sean meca- nismos útiles a la conclusión exitosa del conflicto; no descartó la necesidad de que los pueblos participen de forma directa en La Habana, por ejemplo, mediante la Consulta Previa.

\section{LA CONSULTA PREVIA}

La Consulta Previa, como mecanismo de participación y derecho fundamental, surgió de la discusión en torno a los derechos étnicos en el ámbito internacional, y es el resultado de la lucha protagonizada por el movimiento indígena. Este movimiento se basó en discursos coloniales e indigenistas, que se transformaron en prácticas de resistencia y acciones de liberación que llegarían a incidir en la creación de instrumentos internacionales de protección de derechos étnicos (Rodríguez-Piñero, 2006). Estos instrumentos trascienden la simple protección de los derechos humanos, pues buscan defender los derechos a su identidad y a su libertad de autogobernarse, mediante la creaciòn de mecanismos que les permitan participar en la toma de decisiones sobre los temas que les conciernen.

El Convenio 169 de la olT se convirtió en un instrumento internacional de gran trascendencia por introducir la Consulta Previa como mecanismo orientado a permitir la participación de los pueblos en las decisiones que los afecten. Concretamente, los arts. 6 y 7 determinan que dicho mecanismo se fundamenta en el derecho de todos los pueblos a decidir sobre las medidas susceptibles de afectarles, así como en la facultad de estos de determinar sus prioridades respecto al modelo de desarrollo que institucio- 
nalmente pretenda ser implementado y las posibles afectaciones que puedan tener lugar sobre sus territorios. La garantía y protección de la autodeterminación es uno de los principales objetivos que se pretendió ubicar como fundamento de esta normatividad, a partir de permitir a los pueblos perseguir su propio proceso de desarroIlo y participar en los asuntos que puedan ser de su interés (Rodríguez, Orduz, Rubiano, Boada y Arias, 2010).

Por su parte, la Corte Interamericana de Derechos Humanos (en adelante Corte IDH), a través de sus sentencias, y como resultado de la competencia reconocida por parte de los Estados pertenecientes a la OEA, dentro de los que se encuentra Colombia, ha aportado criterios útiles a la determinación del derecho a la Consulta Previa. En igual sentido ha contribuido la Comisión Interamericana de Derechos Humanos en los casos que son de su competencia, promoviendo su protección y construyendo los parámetros para el amparo al derecho a la Consulta Previa, teniendo una perspectiva de protección de mayores alcances, en contraste con los que se le otorgan a este derecho en las legislaciones nacionales.

En los casos Pueblo indígena Kichwa de Sarayaku vs. Ecuador y Pueblo Saramaku vs. Surinam, la Corte IDH ha mencionado que la obligación de consulta “además de constituir una norma convencional, es también un principio general de Derecho Internacional" y un mecanismo de participación cuya finalidad principal es garantizar la subsistencia de los pueblos indígenas y tribales del mundo. Igualmente, señala que la garantía de este derecho es una tarea que corresponde principalmente al Estado y este no la puede delegar o alegar la dificultad que implica su implementación para evadir su cumplimiento (caso Pueblo indígena Kichwa de Sarayaku vs. Ecuador).

Adicional a lo anterior, dicha Corte afirma la imposibilidad de fijar un único procedimiento para hacer efectivo el mecanismo de participación debido a las diferencias que surgen de un pueblo a otro, de ahí la necesidad de realizar procesos de consulta especiales, diferenciados y culturalmente aceptables (caso Pueblo indígena Kichwa de Sarayaku vs. Ecuador).

Para la Corte IDH la obligación de practicar la Consulta Previa se origina cuando "se vayan a afectar determinados intereses de las comunidades y pueblos indígenas", particularmente en dos escenarios: el desarrollo de un plan de exploración y explotación de recursos naturales en territorios habitados por pueblos indígenas y tribales, y en la expedición de una medida legislativa que amenace con afectarles. Estos escenarios se definirán como generadores de la obligación de aplicar la Consulta Previa, en cabeza de cada Estado, con base en la configuración del criterio de potencial afectación en contra, o incluso a favor, de los pueblos indígenas y tribales (caso Pueblo indígena Kichwa de Sarayaku vs. Ecuador).

La afectación o riesgo del cual se parte para determinar que existe la obligación de realizar la Consulta Previa ha de definirse con la intervención directa de los propios integrantes de los 
pueblos étnicos, pues, como lo afirma la Corte IDH, son ellos los más indicados para establecer la magnitud de los riesgos y afectaciones en sus derechos más trascendentales, y solo así se podrá garantizar la obtención de un consentimiento libre, previo e informado.

Es de resaltar también el énfasis que hace la Corte IDH en el carácter previo de la consulta en cualquiera de los escenarios mencionados anteriormente y la precisión que hace en materia de proyectos de explotación de recursos naturales, cuando aclara que la licencia ambiental no puede ser otorgada sin que se haya practicado la Consulta Previa con antelación (caso del pueblo Saramaka vs. Surinam).

Por último, aclara la Corte IDH que la finalidad que se ha de perseguir al practicar la Consulta Previa no es otra que la de llegar a un acuerdo o lograr el consentimiento acerca de las medidas propuestas, pues el mecanismo no debe agotarse en un simple trámite formal, sino que debe efectuarse como un verdadero instrumento de participación y de consenso (caso del pueblo Saramaka vs. Surinam).

Ahora bien, estos criterios del derecho internacional, resultado de la interpretación de instrumentos de carácter igualmente internacionales, hacen parte de la legislación colombiana y obligan al Estado a cumplirlos en virtud del bloque de constitucionalidad, haciendo de la Constitución Política un texto viviente que se modifica y amplía en la protección de los derechos humanos paulatinamente (Uprimny, 2005).
Es así como el Convenio 169 de la olт hace parte del bloque de constitucionalidad a partir de su ratificación por el Gobierno colombiano a través de la Ley 21 de 1991. Por su parte, la Declaración de las Naciones Unidas sobre los derechos de los pueblos indígenas (DNUDPI), pese a ser reconocida a nivel internacional como una mera recomendación, desarrolla preceptos consagrados en el Convenio y algunos derechos fundamentales de los pueblos, estableciendo parámetros vinculantes que deben ser tenidos en cuenta en el desarrollo de políticas públicas y en la toma de decisiones en el ámbito interno.

La Constitución Política de 1991 consagró en su artículo 330 la obligación del Estado colombiano de garantizar la Consulta Previa frente a casos de explotación de recursos naturales en territorios colectivos. A pesar de esto, no se ha dado un desarrollo normativo libre de inconvenientes en lo concerniente a la legitimidad y a vacíos que, a juicio de la Corte Constitucional: “quebranta[n] el espíritu del Convenio 169 de la oІт". Dentro de la normatividad vigente se encuentra la Ley 70 de 1993, el Decreto 1396 de 1996, el Decreto 1320 de 1998, el Decreto 2613 de 2013 y por último la Directiva Presidencial n. ${ }^{\circ} 10$ de 2013, que deben ser analizados teniendo en cuenta que a pesar de su materia, omitieron la Consulta Previa al momento de ser promulgados.

A su vez, la jurisprudencia constitucional ha desarrollado el derecho a la Consulta Previa adhiriéndose en algunas ocasiones a la normatividad y jurisprudencia internacional. En ese sen- 
tido afirmó en la sentencia C-187/2011² que tanto las medidas administrativas como legislativas, y dentro de estas últimas incluyendo las leyes aprobatorias de los tratados internacionales e incluso reformas constitucionales, han de ser consultadas previamente con los pueblos étnicos.

Previamente, a través la sentencia SU039/1997, la Corte había afirmado que la Consulta Previa era un mecanismo de participación y también un derecho fundamental ligado a la subsistencia de los grupos étnicos, lo que hace que deba buscarse un equilibrio entre el desarrollo económico del país y la subsistencia y protección de los pueblos étnicos; sin embargo, agregó que aunque se trata de un mecanismo de participación, la entidad administrativa que en representación del Estado adelante la consulta, no se encuentra obligada a acatar la decisión tomada por los pueblos, y en caso de desacuerdo, la decisión de la administración debe estar desprovista de arbitrariedad y autoritarismo, debe ser razonable, objetiva y proporcionada a la finalidad constitucional que le exige al Estado la protección de la identidad social, cultural y económica del pueblo indígena o tribal.

Más adelante, en la sentencia C-030/2008 la Corte señaló que uno de los parámetros para identificar las medidas legislativas susceptibles de consulta es la relación que exista entre estas y las materias reguladas por el Convenio 169 de

2 Corte Constitucional colombiana, sentencia C-187 del 16 de marzo de 2011. Magistrado ponente: Humberto Antonio Sierra Porto. la olt, pues considera que no toda medida que se relacione con los pueblos étnicos del país debe pasar por consulta previamente, sino aquellas que constituyan una afectación directa, ya sea positiva o negativa, la cual se configurará cuando se quebrante el equilibrio de la comunidad.

Para ilustrar mejor este punto, la Corte, en sentencia de C-068 el 2013 planteó:

La jurisprudencia constitucional entiende que existe una afectación directa, entre otras, en las siguientes hipótesis: (i) cuando la medida regula un asunto que, por expresa disposición constitucional, debe estar sometido a participación directa de las comunidades étnicas; (ii) cuando la medida se encuentra estrechamente vinculada con el ethos o identidad étnica de los pueblos indígenas; (iii) cuando el objeto principal de la regulación son una o varias comunidades 0 pueblos tribales o el desarrollo concreto de un derecho previsto en el Convenio 169 de 1989 de la OIT; y (iv) cuando a pesar de tratarse de una medida general, (a) esta tiene mayores efectos en las comunidades indígenas que en el resto de la población, o (b) regula sistemáticamente materias que conforman la identidad de dichas comunidades, por lo que puede generarse o bien una posible afectación, un déficit de protección de sus derechos o una omisión legislativa relativa que las discrimine.

En cuanto al procedimiento, la Corte ha recalcado la importancia de que la Consulta Previa esté precedida de un trámite preconsultivo que establezca el conducto regular de este mecanis- 
mo bajo los estándares internacionales y nacionales en cada caso. Esta participación debe ser real y efectiva, puesto que según afirmó la misma Corte en la sentencia C-461 de 2008 no se trata de un simple trámite administrativo, sino de garantizar el ejercicio pleno del derecho a la defensa de los pueblos respecto a las medidas adoptadas, o esfuerzos extemporáneos por parte del Gobierno nacional para cumplir con dicho procedimiento.

Por último, en cuanto a los efectos, la Corte ha entendido que cuando no sea posible lograr el acuerdo u obtener el consentimiento, es necesario revisar el caso de manera concreta para asumir las decisiones correspondientes. Por ello, en la sentencia T-693-2011 conceptuó que llegar a un acuerdo debe ser un objetivo importante al realizar la consulta, lo cual no deja de ser conflictivo pues aunque ha dicho que en algunas circunstancias ese consentimiento sí es indispensable, también ha declarado que eso no significa un poder de veto por parte de las comunidades. El alto tribunal ha manifestado que dicho consentimiento es indispensable cuando: las medidas impliquen el traslado o desplazamiento de las comunidades por la obra o el proyecto; estén relacionadas con el almacenamiento o vertimiento de desechos tóxicos en los territorios étnicos; o representen un alto impacto social, cultural y ambiental que ponga en riesgo la existencia misma del pueblo. En ese sentido, el Estado debe promover esfuerzos intensivos por procurar una solución adecuada para los afectados (sentencia T- 6932011).
Por estas aclaraciones, características y alcances jurídicos otorgados al mecanismo de Consulta Previa; por la importancia de la participación de los pueblos étnicos en las decisiones que versen sobre asuntos de trascendental importancia para sus intereses y que amenacen con afectarles positiva o negativamente, así como por su condición de víctimas del conflicto armado ampliamente conocida por el país, surgió la necesidad de plantearse la pregunta sobre su participación en las decisiones de carácter legal que surjan de los diálogos de La Habana, y sobre si es la Consulta Previa el mecanismo idóneo en orden a dar cumplimiento a las obligaciones que tiene el Estado con estos pueblos y la consecución de una verdadera paz.

Lo anterior, sin dejar de lado que en algunos escenarios se critica el que el objetivo de la Consulta Previa de garantizar la autodetermianción de los pueblos, que se persiguió desde los instrumentos internacionales referidos, quedó limitado y eclipsado con su materialización en mecanismos de participación concretos y de poco alcance ante el temor de los Estados frente a lo que podrían Ilegar a significar las reivindicaciones sociales desde el movimiento indígena (Carrillo-González, 2013), generando disputas judiciales en las cuales las Cortes han venido a complejizar su contenido.

Vale aclarar que los mecanismos que deben promoverse para construir escenarios de paz deben garantizar el diálogo entre los diferentes modos de vida y los temas trascendentales que plantean las luchas y reivindicaciones sociales, constituyendo procedimientos por medio de los 
cuales las diferentes formas de vida logran una concertación de (con)vivencia frente a discrepancias concretas e inmediatas.

\section{LA CONSULTA PREVIA EN EL PROCESO DE PAZ, DESDE LA ÓPTICA DE LOS AFECTADOS}

Como ya se ha demostrado, si bien los diferentes representantes de pueblos étnicos consideran necesaria su participación dentro del proceso de paz que se inició con los diálogos entre el Gobierno y las FARC-EP, han sido claros en que la verdadera construcción de paz exige espacios de participación para toda la sociedad, tal como lo han afirmado varios sectores del país. En el caso particular de los pueblos étnicos, la normatividad nacional e internacional establece la obligación en cabeza del Estado de garantizar su participación, cuando se trate de una decisión que amenace con afectarles. Ahora bien, dado que tanto el conflicto como su terminación permiten vislumbrar la existencia de afectaciones, surge como conclusión obligada la necesidad de llevar a cabo un proceso de participación que pueda adelantarse a través del goce efectivo del derecho de Consulta Previa por ser este el elegido para esta población.

Lo que pasa es que el conflicto ha tenido afectaciones diferenciales, y eso no se puede, tanto no se pueden generalizar las afectaciones del conflicto, como tampoco se pueden generalizar las reparaciones en derecho; deben haber marcos particulares que tengan que ver con la normatividad existente, y la consulta previa es una herramienta para ello, mas no significa que sea un instrumento que vaya a trancar o se vuelva un obstáculo para avanzar hacia la paz y la reconciliación, hay que tener en cuenta los avances en la particularidad y la diferencia, la afectación diferencial y, por su puesto, en la reparación (Juvenal Arrieta).

Así lo manifiestan también otros líderes indígenas entrevistados, considerando todos los puntos de la agenda susceptibles de afectarlos directamente. Por ejemplo, Axcan Duque, representante afrocolombiano, observó cómo los posibles acuerdos podrían resultar en otras afectaciones:

Hay varios puntos, la política antidrogas en el país debe cambiar, la participación política también, recordemos que nuestros territorios van. Iban las avionetas, fumigaban o nos sometían los erradicadores manuales, que también estaban haciendo un trabajo y demás, pero la Corte Constitucional muy sabiamente indicó que tanto fumigación como erradicación cuando se trata de territorios ancestralmente habitados o territorios étnicos debían consultarse, entonces la política antidrogas debe consultarse. Segundo, la participación, la participación política, las mayores víctimas en este país han sido los grupos étnicos no solo por el conflicto social, político y armado, también por la exclusión social y la brecha histórica que se ha dado (...) En territorio, se requiere la reforma agraria y esa reforma agraria de nuestros territorios, recuerdan, digo recuerdan pero es una forma de hablar, es que nuestros territorios no tienen derecho a saneamiento los territorios indígenas sí, y en nuestros territorios fueron muchas colonos bajo la estrategia paramilitar. Si ustedes ven Nigua y Guamiandó y Curbaradó se nos metieron un pocotón de colonos, pero a 
esos colonos no se les puede quitar porque ya tienen un derecho real de posesión y demás sobre la tierra, pero entonces si existiera la política de saneamiento para los territorios de comunidades negras lo podríamos hacer.

Aún así, existe preocupación sobre el momento en que debería realizarse dicha consulta. La mayoría de entrevistados opinaron que debería realizarse una vez se termine la etapa de negociación y se inicie la etapa de implementación, pues es en esta donde se vislumbran los riesgos de afectación:

después viene la parte de implementación de los acuerdos, que ahí es dónde venimos a jugar nosotros, que las FARC y el gobierno nos expliquen a nosotros cómo van a implementar estos acuerdos, ¿lo van hacer bajo qué figura?, ¿plan de desarrollo nacional?, ¿con Conpes?, ¿con presupuesto?, ¿cómo lo van a implementar? Segundo: ¿qué van a implementar, proyectos productivos?, ¿asociaciones cooperativas? No sabemos, y si fueran proyectos a gran escala dónde vamos a estar involucrados nosotros, es ahí donde juega la consulta previa (Feliciano Valencia).

Así las cosas, los entrevistados vieron que la aplicación de la Consulta Previa tendría trascendencia en la segunda etapa del proceso, pues lo que se espera de estas negociaciones entre Gobierno y FARC, es que surjan nuevas leyes, nuevas reglas y nuevos proyectos que permitan concretar un cambio social, que eventualmente debería terminar en la paz. Empero, para que esto sea posible, los mecanismos de participación no se deberían limitar a cumplir su función en la garantía de derechos, por el contrario, los líderes de los diferentes pueblos coinciden en que al permitir la participación de los afectados en la creación de las normas se estaría dando un paso adelante en la construcción de la paz.

Pero la consulta previa no solo tendría un rol de garantía, también sería un mecanismo que permitiría la construcción de paz, pues creemos que la consulta previa sí es un instrumento importante en la reconciliación y en la posibilidad de avanzar hacia la paz de los colombianos, porque estarían muchas cosas en la mesa, tu sabes que en la consulta se interpelan muchas cosas, se interpela el modelo de desarrollo económico, se interpela el ejercicio de la participación política, se interpela el ejercicio de los derechos civiles, se interpela el ejercicio de los derechos laborales, se interpelan muchas cosas que tienen que ver incluso con el consentimiento (Juvenal Arrieta).

Pese a resultar evidente la obligatoriedad de la Consulta Previa en este escenario, y a que algunos la consideran una herramienta para la construcción de paz, otros tienen varias reservas sobre su efectividad y su procedencia. Desde el punto de vista de la academia, tuvimos oportunidad de entrevistar a la profesora Clara Henao, quien considera que la Consulta Previa no es un mecanismo de participación que se deba aplicar a un determinado grupo de sujetos o a un pueblo en especial, sino a toda la sociedad, es decir, que toda la sociedad debe tener la oportunidad de participar en las decisiones que la afectan, en cumplimiento de la descentralización propuesta por la Constitución. En su concepto, la garantía de los derechos de los pueblos étnicos del país no es un tema de Consulta 
Previa sino de igualdad y de dignidad. Es más, cree que la Consulta Previa constituye más un perjuicio que un beneficio y cuestiona el que la participación de estos pueblos se dé a través de las organizaciones, las cuales no representan efectivamente a cada uno de los integrantes. Al respecto manifiestó:

se trata es de consultar a la diversidad, ellos no son homogéneos, o sea, yo no puedo decir, los indios, y entonces porque alguien es indio dice yo hablo por todos los indios, no, como así, dentro de los mismos grupos indígenas existe una diversidad demasiado amplia... las leyes y las normas no están surgiendo de las necesidades y de los proyectos jurídicos propios de cada región, sino que están es surgiendo de una necesidad de hacer una plataforma de desarrollo, lo que se está negociando en este momento en términos de leyes no es para favorecer a la población, es para permitir la entrada de unas propuestas corporativas, de globalización, neoliberales, aquí no estamos negociando realmente para nada cómo mejorar el nivel de vida de nadie, entonces la medidas digamos jurídicas y legislativas y administrativas no deben ser consultadas solamente con las comunidades indígenas y negras, pues, con los grupos étnicos que es un derecho que ellos tienen por ley 21 , sino todos los grupos.

En este orden de ideas, algunos líderes Ilegaron a manifestar cierto grado de miedo e inconformidad con los parámetros bajo los cuales se adelanta la consulta. Entre estos, Ben Hur Teteye manifestó:

En un escenario en que estas autoridades tuvieran ingreso al escenario que se desarrolla en La Habana y no hubiese un acuerdo entre nuestras peticiones y los otros actores, no pasaría nada porque la institución gubernamental sigue su camino y por otro lado el movimiento sigue su objetivo, lo que sí puede suceder es que a pesar de los desacuerdos se logren entablar nuevas vías de participación.

Las preocupaciones no son injustificadas: por un lado, es cierto que el consentimiento no es un requisito para validar el proceso de Consulta Previa, lo que implica que la obligación legal de implementarla podría agotarse al llevar a cabo el proceso sin que este tenga verdaderas implicaciones en las decisiones finales; por otro lado, y lo que es aún más preocupante, es la perspectiva bajo la cual el Estado ve la Consulta Previa. En la entrevista con el director de Consulta Previa del Ministerio del Interior, Álvaro Echeverri, frente a la labor de la Dirección a su cargo manifestó que, pese a existir la imposibilidad de contestar las preguntas concernientes al proceso de paz por ser este un tema de orden público, sobre el que los únicos que podrían expresarse son el Presidente, el Ministro de Defensa o el Ministro del Interior, sí se referiría al mecanismo de Consulta Previa:

la función de consulta previa es eminentemente una función de carácter arbitral, es de carácter notarial, nosotros lo único que hacemos es promover los mecanismos, las herramientas necesarias, para que dos partes en un diálogo se pongan de acuerdo, o para certificar notarialmente que no obstante haberse agotado las instancias, no hubo posibilidad de que se llegara a un acuerdo entre las partes. Más allá nosotros no podemos entrar en ningún escenario, pues no le corresponde al árbitro hablar de las tácticas utilizadas por los equipos, ni le 
corresponde al árbitro definir cuáles son las alineaciones, ni le corresponde al árbitro hacer comentarios posteriores sobre cómo vio el partido o cuál equipo jugó mejor.

Afirmación que deja un vacío frente al papel que esta entidad debe desempeñar como garante cuando una de las partes es el mismo Gobierno nacional.

\section{CONCLUSIONES}

Para los pueblos étnicos el proceso de paz implica un esfuerzo complejo que va más allá de la dejación de armas, incluso más allá de las pretensiones de la agenda de La Habana, pues se plantea la necesidad de hablar de procesos de reconciliación y de una reparación integral y transformadora que no solo sea de carácter económico, si en realidad se quiere garantizar una verdadera construcción de paz. Ahora bien, es claro que la realización de estos propósitos exige la práctica de mecanismos de participación que involucren a los afectados/as.

Como resultado del análisis de la Consulta Previa como mecanismo de participación y derecho fundamental, que se activa ante situaciones que producen una afectación positiva o negativa para los pueblos étnicos, y teniendo en cuenta el escenario de negociación entre el Gobierno y las FARC-EP, se puede concluir que independiente del tema sobre el cual se tomen decisiones, es necesaria la participación de los pueblos en el proceso y en la implementación de los acuerdos que de allí surjan, por cuanto se trata de un deber del Estado colombiano, más aún si el propósito coincide con el logro de la paz y estos estarían sometidos al exterminio físico y cultural, a pesar de las declaratorias de diversidad étnica y cultural de nuestra nación. Es innegable que la paz debe incluir el final de las formas históricas de exclusión en contra de los pueblos étnicos del país, ya que de otro modo no habría una conciliación prolongada.

Sin embargo, las exigencias que trae consigo la práctica de la Consulta Previa implican grandes retos para el Estado colombiano en el marco de un eventual proceso de paz, y no son pocos los que desconfían del mecanismo, principalmente por resultar inadecuado para la verdadera garantía de derechos de estos pueblos y su papel como constructores de paz dentro de la nación, y más aún cuando el Estado parece dispuesto a asumir una postura arbitral y a dejar de lado su obligación de proteger los derechos humanos bajo los preceptos de un Estado social de Derecho que busca una reparación integral y la superación del conflicto armado y sus factores vinculados y subyacentes.

Aún así y pese a la desconfianza en torno a la Consulta Previa, producto de diferentes factores que han entorpecido su práctica, los pueblos étnicos necesitan de este mecanismo para su protección efectiva sin que sea visto como un obstáculo o un mero trámite administrativo para el Estado. Vislumbrando en el horizonte la luz tenue de una posible paz, queda la incertidumbre de cómo el Gobierno y las FAR-EP ejecutarán lo dialogado, sin que en este paso se excluya a una parte de la población que desde sus raíces tiene una noción de vida en comunidad basada 
en el respeto y la convivencia pacífica, así como con preservar el equilibrio y armonía entre todos los seres que integran la naturaleza, y que ante el conflicto armado interno siempre ha encabezado la lucha por la construcción de una paz que les ha sido ajena históricamente.

El Grupo COPAL cree que para que haya paz es requisito irrenunciable el que exista un verdadero derecho a la libre autodeterminación de los pueblos etnicos; que estos ciudadanos puedan decidir con libertad, autonomía y sin ataduras sus rumbos, formas y procesos ajenos a sus designios; en fin, que puedan tener un diálogo con sus conciudadanos en igualdad de condiciones. Ahora bien, que lo anterior sea una realidad a través del mecanismo de Consulta Previa o no, es cuestión que les compete decidir a ellos mismos.

\section{Referencias}

Carrillo González, D. M. (2013). Jurisdicción especial indígena: ¿mecanismo de fortalecimiento o debilitamiento del derecho a la libre determinación de los pueblos indígenas? Bogotá: Universidad Nacional de Colombia. Facultad de Derecho y Ciencias Políticas y Sociales.

Consultoría para los Derechos Humanos y el Desplazamiento. (2012). Desplazamiento creciente y crisis humanitaria invisibilizada. Obtenido de CODHES: http://www.acnur.org/ t3/uploads/media/CODHES_Informa_79_ Desplazamiento_creciente_y_crisis_humanitaria_invisibilizada_Marzo_2012.pdf?view=1
Corbetta, P. (2007). Metodología y técnicas de investigación social. Madrid: McGraw-Hill/Interamericana.

Corte Constitucional de Colombia. Auto 382 de 2010. Sala especial de seguimiento a la sentencia T-025 de 2004, integrada por los magistrados Juan Carlos Henao Pérez, Nilson Pinilla Pinilla y Luis Ernesto Vargas Silva.

Corte Constitucional de Colombia. Sentencia C-030 del 23 de enero de 2008. M. P.: Rodrigo Escobar Gil.

Corte Constitucional de Colombia. Sentencia C-068 del 13 de febrero de 2013. M. P.: Luis Guillermo Guerrero Pérez.

Corte Constitucional de Colombia. Sentencia C-175 del 18 de marzo de 2009. M. P.: Luis Ernesto Vargas Silva.

Corte Constitucional de Colombia. Sentencia C-317 del 3 de mayo de 2012. M. P.: María Victoria Calle Correa.

Corte Constitucional de Colombia. Sentencia SU-039 del 3 de febrero de 1997. M. P.: Antonio Barrera Carbonell.

Corte Constitucional de Colombia. Sentencia T-693 del 23 de septiembre de 2011. M. P.: Jorge Ignacio Pretelt Chaljub.

Corte Interamericana de Derechos Humanos. Caso Pueblo indígena kichwa de Sarayaku vs. Ecuador. 27 de junio de 2012. 
Corte Interamericana de Derechos Humanos. Caso Pueblo Saramaka vs. Surinam. 28 de noviembre de 2007.

Organización Nacional Indígena de Colombia. (2012). Por la defensa, respeto y exigibilidad de los derechos de los pueblos indígenas en colombia. Obtenido de onIc: http:// cms.onic.org.co/wp-content/uploads/downloads/2012/10/Reporte-Enero-Septiembre_2012.pdf

Organización Nacional Indígena de Colombia. Autoridad Nacional de Gobierno Indígena. (14 de marzo de 2013). Pueblos Indígenas en riesgo de exterminio físico y cultural: caso Colombia. Intervención en el 147 periodo de sesiones de la Comisión Interamericana de Derechos Humanos. Recuperada de: http:// cms.onic.org.co/wp-content/uploads/downloads/2013/03/Pueblos-Ind\%C3\%ADgenasen-riesgo-de-exterminio-ONIC-AUDIENCIA14-DE-MARZO-WASHIGTON1.pdf

Organización Nacional Indígena de Colombia. (2014). Informe 2014 de la Consejería de Derechos de los Pueblos Indígenas, Derechos Humanos, DIH y Paz. Recuperado de: http://cms.onic.org.co/wp-content/ uploads/2014/09/Informe-Anual-2014-Derechos-Humanos-ONIC.pdf

Restrepo, J. y Aponte, D. (Eds.). (2009). Guerra y violencias en Colombia: herramientas e interpretaciones. Bogotá: Editorial Pontificia Universidad Javeriana.
Rodríguez Garavito, C., Alfonso Sierra, T. y Cavelier Adarve, I. (2009). Raza y derechos humanos en Colombia: primer informe sobre discriminación racial y derechos de la población afrocolombiana. Bogotá: Ediciones Uniandes.

Rodríguez Garavito, C., Orduz Salinas, N., Rubiano, S., Boada, S., y Arias, F. (2010). Pueblos indígenas y desplazamiento forzado. Evaluación del cumplimiento del auto 004 de la Corte Constitucional Colombiana. Programa de Justicia Global y Derechos Humanos. Bogotá: Universidad de los Andes.

Rodríguez-Piñero, L. (2006). Indigenous Peoples, Postcolonialism and International Law: The ILO Regime (1919-1989). Oxford: Oxford University Press.

Semana.com. (8 de febrero de 2014). Seis miIlones de víctimas deja el conflicto en Colombia. Obtenido de: http://www.semana.com/ nacion/articulo/victimas-del-conflicto-armado-en-colombia/376494-3

Uprimny Yepes, R. (2005). El bloque de constitucionalidad en Colombia. Un análisis jurisprudencial y un ensayo de sistematización doctrinal. En D. O’Donnell, I. M. Uprimny, A. Valencia Villa y M. J. Díaz Granados, Compilación de jurisprudencia y doctrina nacional e internacional. Bogotá: Oficina en Colombia del Alto Comisionado de las Naciones Unidas para los Derechos Humanos. 